\title{
Original Research \\ Cost evaluation of therapeutic drug monitoring of gentamicin at a teaching hospital in Malaysia
}

\author{
Mohamed Izham IBRAHIM, Hisham Elhag ABDELRAHIM, Ab Fatah AB RAHMAN.
}

Received (first version): 3-Oct-2013

Accepted: 8-Mar-2014

\begin{abstract}
${ }^{*}$
Background: Therapeutic drug monitoring (TDM) makes use of serum drug concentrations as an adjunct to decision-making. Preliminary data in our hospital showed that approximately one-fifth of all drugs monitored by TDM service were gentamicin.

Objective: In this study, we evaluated the costs associated with providing the service in patients with bronchopneumonia and treated with gentamicin. Methods: We retrospectively collected data from medical records of patients admitted to the Hospital Universiti Sains Malaysia over a 5-year period. These patients were diagnosed with bronchopneumonia and were on gentamicin as part of their treatment. Five hospitalisation costs were calculated; (i) cost of laboratory and clinical investigations, (ii) cost associated with each gentamicin dose, (iii) fixed and operating costs of TDM service, (iv) cost of providing medical care, and (v) cost of hospital stay during gentamicin treatment.

Results: There were 1920 patients admitted with bronchopneumonia of which $67(3.5 \%)$ had TDM service for gentamicin. Seventy-three percent (49/67) patients were eligible for final analysis. The duration of gentamicin therapy ranged from 3 to 15 days. The cost of providing one gentamicin assay was MYR25, and the average cost of TDM service for each patient was MYR104. The average total hospitalisation cost during gentamicin treatment for each patient was MYR442 (1EUR approx. MYR4.02).

Conclusion: Based on the hospital perspective, in patients with bronchopneumonia and treated with gentamicin, the provision of TDM service contributes to less than $25 \%$ of the total cost of hospitalization.
\end{abstract}

Keywords: Drug Monitoring; Costs and Cost Analysis; Pharmacy Service, Hospital; Gentamicins;

Bronchopneumonia; Malaysia

\section{INTRODUCTION}

Therapeutic drug monitoring (TDM) is defined as the measurement made in the laboratory of a parameter that, with appropriate interpretation, will directly influence prescribing procedures. ${ }^{1}$ TDM service comprises of measuring drug concentration, generating individual patient's pharmacokinetic data, and interpreting such data together with the patient's clinical conditions to optimise his/her drug

\footnotetext{
"Mohamed Izham Mohamed IBRAHIM. PhD. College of Pharmacy, Qatar University. Doha (Qatar). mohamedizham@qu.edu.qa

Hisham Elhag Ahmed ABDELRAHIM. MSc. Director of Health Promotion Department, \& Khartoum Medicines Information Centre (KhMIC), General Directorate of Pharmacy, Ministry of Health. Khartoum (Sudan). hishamhag@yahoo.com

Ab Fatah AB RAHMAN. PharmD. School of

Pharmaceutical Sciences, Universiti Sains Malaysia. Pinang (Malaysia). abfatahmy@yahoo.com
}

therapy. Since the early 1970 s, it has been used to individualise drug therapy in patients on selected drugs with a narrow therapeutic index and wide pharmacokinetic variability like antiepileptics, aminoglycosides, and digoxin. TDM is also useful in cases where patient drug compliance is questionable, and where drug toxicity is suspected.

To be meaningful, drug concentration must be available in a timely manner and commercially available immunoassays offer the most convenient method of drug analysis. However, the cost of performing drug assays especially in a developing country puts a constraint on a hospital's budget. ${ }^{2,3}$ In this era of cost-conscious healthcare environment, the pressure is on TDM practitioners to maintain a cost-effective service. However, studies evaluating costs associated with providing TDM services are not that common. Earlier report shows that out of 247 TDM studies conducted over a 20-year period, only $8 \%$ incorporated pharmacoeconomic analysis. ${ }^{4}$ Many of these pharmacoeconomic studies of TDM were conducted on aminoglycosides antibiotics. ${ }^{5}$ In a more recent analysis, Touw et al. $^{6}$ concluded TDM is costeffective only for aminoglycosides, whereas TDM of vancomycin is cost-effective in selected patient populations only. Although TDM of other drugs is recommended, data on cost-effectiveness is still lacking.

At Hospital Universiti Sains Malaysia (HUSM), TDM of aminoglycosides was started in the early 1980s. Early reports have shown that it had positive impacts on therapeutic concentrations and on prescribing of gentamicin in our setting but data on cost evaluation of the service are not available. ${ }^{7,8}$ Preliminary TDM data from 2000 to 2005 showed that gentamicin represented $20 \%$ of all drugs monitored by the TDM service. In this article, we report the findings of a cost-evaluation of TDM service in patients diagnosed with bronchopneumonia and on gentamicin treatment.

\section{METHODS}

\section{Study design}

This study employed a retrospective research design to perform a pharmacoeconomic evaluation of a hospital TDM service at the Hospital Universiti Sains Malaysia (HUSM). Data were collected retrospectively from medical records of eligible patients. The study was performed based on the hospital and health care provider perspectives. The study was performed at the HUSM, Malaysia. Data collection started in January and ended in March 
2006. All aspects of the study protocol, including access to and use of the patient clinical information, were approved by the HUSM Administration Committee, chaired by the director of the hospital (Reference:HUSM/11/010).

\section{Population and sampling}

A computer generated list based on diagnosis code was provided by the Medical Records Office of HUSM. Medical records of adult patients admitted to the hospital from 2001 to 2005 with a diagnosis of bronchopneumonia infection were identified. The inclusion criteria for review were patients at least 18 years of age, diagnosed as bronchopneumonia with proven or suspected gram-negative infection, admitted to the hospital for at least 72 hours, treated with gentamicin for at least 72 hours and had at least one gentamicin assay measurement. ${ }^{9,10}$ Patients with existing renal impairment, who had history of aminoglycosides toxicity, with duration of gentamicin therapy less than 72 hours, or who received concurrent treatment with known nephrotoxic drugs were excluded. ${ }^{11,12}$ In addition, patients in whom gentamicin TDM service was requested after 72 hours of starting gentamicin treatment were excluded. Records of patients eligible for the study were then searched in the TDM database for details on gentamicin monitoring.

\section{Data collection procedure}

Data were collected for the following: patient demographics, duration of hospital stay, concomitant disease state, clinical and laboratory investigations, and the total number of serum concentration measurements done. The duration of gentamicin therapy, dosage regimen, duration of each regimen, concurrent antibiotics and medications were also determined. Nephrotoxicity was defined as an increase in serum creatinine by $50 \%$ of its baseline. ${ }^{11,13}$ Duration of gentamicin therapy was calculated from duration the patient was hospitalised during gentamicin therapy.

\section{Data management and analysis}

Data on cost were obtained from the administrative office and bursary department of HUSM. TDM and gentamicin therapy data were obtained from the TDM laboratory and Department of Pharmacy. Five different hospitalisation costs were calculated for each patient; (i) cost of laboratory and clinical investigations during gentamicin therapy. These laboratory tests included blood profile, blood/sputum cultures, serum creatinine, electrolyte profile and chest X-rays. The total cost was calculated based on the hospital fee for each specific test multiplied by the number of tests done for each patient; (ii) cost associated with each gentamicin dose, which included the cost of gentamicin and disposables like syringes and alcohol swab; (iii) cost of providing TDM service, which included fixed costs (TDM laboratory space and laboratory equipment) and operating costs (i.e. time spent by the TDM team, reagents and consumables). Cost of TDM laboratory space was calculated by multiplying the area of the laboratory in square feet by the cost per square foot. TDM laboratory equipment included TDM instruments and furniture. The cost of laboratory equipment was calculated by subtracting the depreciated amount from the cost upon purchase by using $3 \%$ as a depreciation percentage rate. ${ }^{14}$ Gentamicin represented $20 \%$ of all tests performed, therefore, both the laboratory space and laboratory equipments costs were multiplied by 0.20 . The total operating cost was the summation of cost of reagents and consumables, and cost associated with the contribution of the TDM team. TDM team included the nurse, laboratory technician, and pharmacist. In our setting, nurses are responsible for blood collection, and the time spent for this activity was taken into consideration. Calculation of costs associated with the involvement of each of these health professionals was based on their basic monthly salaries and time spent performing the tasks; (iv) cost of providing medical care by doctors was calculated after taking into account their basic monthly salaries and the average duration spent for each patient visit. For nursing care time, it was determined from the time spent for performing clinical investigations such as measuring body temperature, and the time spent associated with the administration of gentamicin dose. Both costs were combined as cost of providing medical/nursing care; ( $\mathrm{v}$ ) cost of hospital stay during gentamicin treatment included the hospital room charges, and cost associated with non-medical services in the ward for the entire duration of gentamicin therapy.

For the data obtained, we performed descriptive statistics and presented as mean (SD), median, frequency and percentage where appropriate.

\section{RESULTS}

There were 1,920 patients admitted to the hospital for chest infection and bronchopneumonia over the five-year period. Figure 1 shows how selection was made based on the selection criteria. Sixty-seven patients were diagnosed with bronchopneumonia and at the same time provided with the TDM service for gentamicin. Only 49 patients were eligible for further analysis. Patients age ranged from 18 to 77 years old (median 42 years). Thirty-seven patients were male and 12 patients female. Ninety-six percent of patients were Malay. The study was not able to report how many of the 1,920 patients were on gentamicin.

All patients received conventional multiple daily dosing of gentamicin. The average dose was found to be $241.8(S D=57.5) \mathrm{mg} /$ day (range: 120 to 375 $\mathrm{mg} /$ day). All patients had other concurrent medical problems (eg. chronic obstructive pulmonary disease, diabetes, sepsis, hypertension). All except one patient were on concurrent antibiotics (e.g. ceftazidime, cloxacillin, erythromycin, penicillin G). Serum creatinine was measured in all patients before gentamicin therapy but this parameter was only available in 27 patients after therapy. Of these, none experienced an increase in serum creatinine over $50 \%$ from baseline (range: $-42 \%$ to $45 \%$ ). The mean duration of gentamicin therapy was 7.8 $(S D=2.9)$ days (range: 3 to 15 days). 


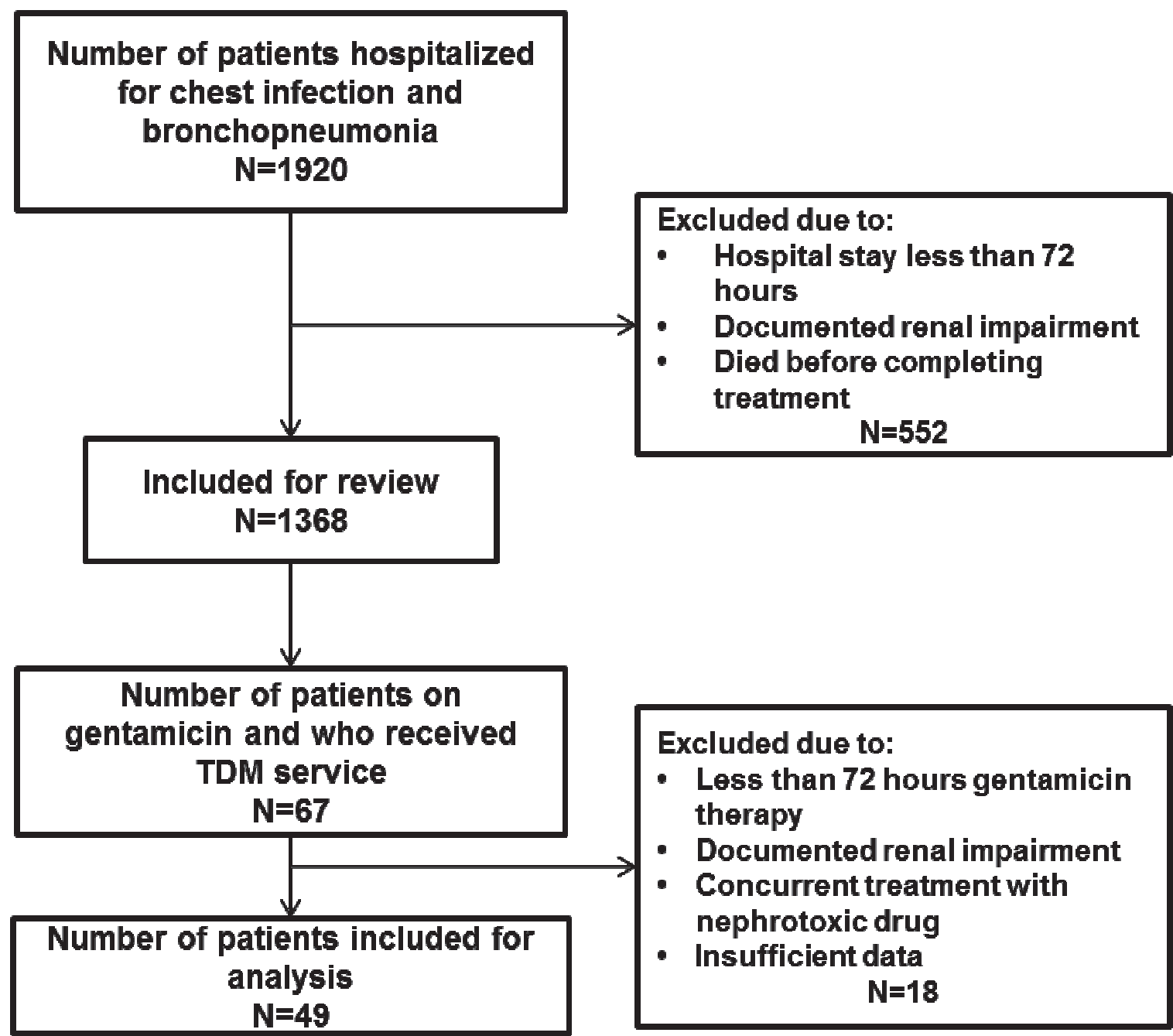

\section{Cost analysis}

Table 1 shows the distribution of costs associated with each of the components that made up the total cost of hospitalisation. The total cost of laboratory/clinical investigations and X-rays ranked the highest compared to other hospitalisation costs [Range: MYR42.00 to MYR336.00; (1 EUR approx. MYR4.02)]. In about $60 \%$ patients, the cost of laboratory/clinical investigations and X-rays was between MYR101.00 and MYR200.00.

The cost of providing TDM service was obtained from fixed and operating costs. Based on data provided by the HUSM development office, the cost of TDM laboratory space was MYR9,800. The total cost of TDM equipment was estimated at MYR50,000. Overall, the total fixed cost was MYR12.90. The costs associated with time spent by the nurse, technician and pharmacist were MYR0.40, MYR0.75 and MYR1.30, respectively. Serum drug concentration was measured using fluorescence polarisation immunoassay (FPIA) technique (Abbott Lab., USA). This assay technique was used throughout the five-year period and there was no change in vendor. Each patient had an average of four gentamicin assays performed (range: 1 to 12 assays). The cost of TDM reagents and consumables associated with one gentamicin assay was found to be MYR10.12. Overall, the total operating costs associated with one gentamicin assay was MYR12.57. Therefore, the total cost of providing TDM service for one gentamicin assay was MYR25.47.

The daily cost of medical/nursing care associated with time spent by doctor and nurse for each patient was found to be MYR6.25. In this cohort of patients, the cost of hospital stay (ie. room charges and nonmedical services) ranged from MYR9.00 to MYR45.00. It ranked the lowest and contributed to about $5 \%$ of the total hospitalisation cost.

\section{DISCUSSION}

TDM is widely available in Malaysia ${ }^{15}$ but this was the first study to look at the cost of providing the service from the hospital perspective. We have chosen to evaluate TDM of gentamicin in patients with bronchopneumonia because preliminary data showed that the most common drug monitored during that period was gentamicin $(20 \%)$, and that among patients treated with gentamicin, the most common infection was bronchopneumonia (27\%). Although nearly 2000 patients were hospitalised for bronchopneumonia over the five-year period, only 49 adult patients satisfied the selection criteria. In this hospital, all patients on gentamicin will be 
Ibrahim MI, Abdelrahim HE, Ab Rahman AF. Cost evaluation of therapeutic drug monitoring of gentamicin at a teaching hospital in Malaysia. Pharmacy Practice 2014 Jan-Mar;12(1):372

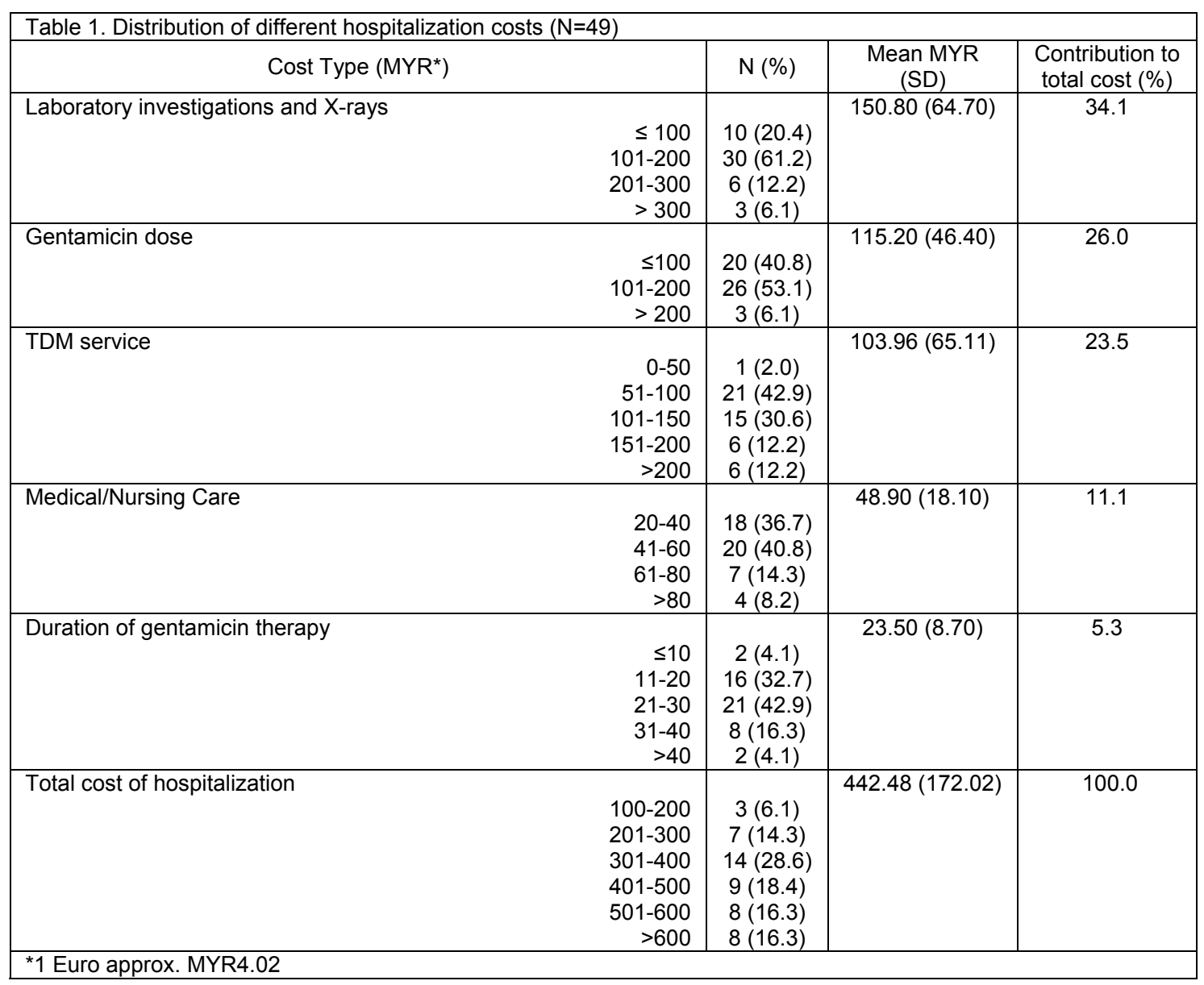

monitored for serum concentrations. Therefore, it could be deduced that only 67 patients received gentamicin during this period.

In this study, we have attempted to quantify the monetary cost associated with the TDM service and how it contributes to the overall cost of hospitalisation during gentamicin therapy. Providing TDM service does not just involve the measurement of drug concentration. It is a multidisciplinary activity. ${ }^{16}$ It requires the collaboration among doctors, nurses, pharmacists and laboratory staff. Therefore, cost calculation of TDM service requires not only placing a value on the existing infrastructures (fixed costs) but also on the contributions of each of these health care staffs (operating costs).

Several factors need to be considered when looking at the fixed cost associated with providing TDM service. As with other laboratories, the TDM laboratory has many other types of equipment, which are not directly related to the measurement of drug concentration but are essential for day-to-day running of the service. Each of these items has a different life expectancy. It is difficult to calculate the cost of each item individually due to its life expectancy. Accordingly, a constant value (i.e. $3 \%$ ) was used as a depreciation rate for all these laboratory equipment. ${ }^{14}$ The application of this depreciation rate reduces the cost of each item depending on the year purchased. Therefore, the calculated cost of the laboratory equipment was found to be less by about $40 \%$ from the purchased cost (i.e. equals to $61 \%$ of the total purchased cost).

For operating cost, it seems that the cost of reagents was substantially higher than cost associated with time spent by the TDM team. Fluorescence polarisation immunoassay (FPIA) technique used to measure drug concentration has a short turnaround time and is easy to run. However, its reagents are costly because Malaysia imports these TDM reagents from the USA through local vendors. Our results show that most patients were hospitalised for an average of about eight days and many had more than one assay measurement. Repeated measurement of TDM assays is a common practice in HUSM and this may contribute to increase in operating cost. HUSM TDM pharmacist often recommends doctors to repeat the assay after 72 hours. The pharmacist might have asked the assay to be repeated in order to ensure that therapeutic concentrations had been achieved based on dosing changes previously made. It is also likely that these repeat assays were carried out on the basis of the clinical status of the patient at the time. ${ }^{17,18}$

The cost associated with administration of gentamicin dose made up $26 \%$ of total hospitalisation cost. In this study, all patients were treated with conventional dosing regimen of gentamicin. Providing multiple daily doses of gentamicin will increase the overall cost because each administration is associated with the use of several consumables. Unlike multiple daily dosing 
regimen, single daily dosing regimen of gentamicin has widely used and has the advantages of lowering the cost of treatment. ${ }^{19}$

Overall, our findings show that the total hospitalisation cost was dominated mostly by cost of imported reagents and consumables (eg. TDM reagents, reagents used for other laboratory investigations, and X-ray films), and cost of drug used (eg. gentamicin). On the other hand, labour costs (eg. TDM team and medical/nursing care) and hospital room charges contributed minimally to the overall cost of hospitalisation. There was a wide variation in the duration of hospitalisation seen among this cohort of patients. However, HUSM only imposed a nominal fee of MYR8.50 per day for inpatients. This may be the reason that cost associated with hospital stay was the lowest among other hospitalisation costs.

In our setting, TDM service constituted about $24 \%$ of the total overall hospitalisation cost for the duration of gentamicin therapy. While labour costs are somewhat similar to other hospitals in the country, the cost associated with TDM assay may be different. Like other immunoassays, TDM assay reagents have expiration dates. Therefore, within that shelf life, there is a higher likelihood that these reagents will not be fully used especially in hospitals with low volume of TDM requests. As a result, the cost associated with the TDM service will increase.

Since this was the first cost-analysis study carried out on TDM service in our setting, the findings could serve as a baseline data for future evaluation. Thus, any increase in cost contribution by the TDM service in the future should be carefully scrutinised. At the same time, we believe that there is still room for improvement to make the service more costeffective. While the price of reagents and consumables is beyond the TDM pharmacist's control, the judicious utilisation of this service like improvement in appropriate indication and sampling should be made a priority.

This study has its limitations. The analysis was conducted only on a small number of patients, which may contribute to bias. Medical records were not fully computerized and might not contain complete data. Therefore, we could not completely rule out the possibility that certain data like laboratory investigations might be missing from the records although the tests were actually done.

\section{CONCLUSIONS}

In patients with bronchopneumonia and treated with gentamicin, the provision of TDM service contributes to less than $25 \%$ total cost of hospitalisation. It is imperative that the service is used judiciously to avoid unnecessary increase in cost in the future.

\section{CONFLICT OF INTEREST}

None to declare.

Funding: Self-funded.

\section{EVALUACIÓN DEL COSTE DE LA MONITORIZACIÓN SÉRICA DE GENTAMICINA EN UN HOSPITAL UNIVERSITARIO DE MALASIA}

\section{RESUMEN}

Antecedentes: La monitorización sérica de medicamentos (TDM) utiliza las concentraciones séricas como un elemento para la toma de decisiones. Los datos preliminares de nuestro hospital indican que aproximadamente un quinto de todos los medicamentos seguidos por TDM fue la gentamicina.

Objetivo: En este estudio evaluamos los costes asociados con la provisión del servicio en pacientes con bronconeumonía y tratados con gentamicina. Métodos: Recogimos retrospectivamente datos de las historias clínicas de los pacientes ingresados en el Hospital Universidad de Sains Malasia durante 5 años. Estos pacientes estaban diagnosticados con bronconeumonía y estaban con gentamicina como parte de su tratamiento. Se calcularon 5 costes de hospitalización: (i) costes del laboratorio y análisis clínicos, (ii) costes asociados con cada dosis de gentamicina, (iii) costes fijos y operativos del servicio de TDM, (iv) coste de proporcionar atención médica, y (v) costes de la hospitalización durante el tratamiento con gentamicina.

Resultados: Hubo un total de 1920 pacientes ingresados con bronconeumonía de los que $67(3,5 \%)$ tuvieron servicio de TDM para la gentamicina. El $73 \%(49 / 67)$ de los pacientes estaban incluidos en el análisis final. La duración del tratamiento de gentamicina osciló entre 3 y 15 días. El coste de realizar cada prueba de gentamicina era de 25 MYR, y el coste medio del servicio de TDM por paciente fue de 104 MYR. El coste medio de hospitalización por paciente durante el tratamiento con gentamicina fue de 442 MYR (1 EUR aprox. 4.02 MYR). Conclusión: Desde la perspectiva hospitalaria, los pacientes con bronconeumonía tratados con gentamicina, la provisión del servicio de TDM contribuye menos del $25 \%$ al total de costes de hospitalización.

Palabras clave: Monitorización de medicamentos; Costes y Análisis de Costes; Servicio de Farmacia Hospitalario; Gentamicinas; Bronconeumonía; Malasia

1. Watson I, Potter J, Yatscoff R, Fraser A, Himberg JJ, Wenk M. Definition of therapeutic drug monitoring. Ther Drug Monit. 1997;19(2):125

2. Gogtay NJ, Kshirsagar NA, Dalvi SS. Therapeutic drug monitoring in a developing country: an overview. Br J Clin Pharmacol. 2001;52(Suppl 1):103S-108S.

3. Setiabudy R. Therapeutic drug monitoring: focus on conditions in Indonesia. Acta Med Indones. 2011;43(3):208-211. 
4. Schumacher GE, Barr JT. Economic and outcome issues for therapeutic drug monitoring in medicine. Ther Drug Monit. 1998;20(5):539-542.

5. Destache CJ. Use of therapeutic drug monitoring in pharmacoeconomics. Ther Drug Monit. 1993;15(6):608-610.

6. Touw DJ, Neef C, Thomson AH, Vinks AA; Cost-Effectiveness of Therapeutic Drug Monitoring Committee of the International Association for Therapeutic Drug Monitoring and Clinical Toxicology. Cost-effectiveness of therapeutic drug monitoring: a systematic review. Ther Drug Monit. 2005;27(1):10-17.

7. Ismail R, Sarriff A, Abdul Rahman AF. Therapeutic drug monitoring for gentamicin in Hospital Universiti Sains Malaysia. Med J Malaysia. 1990;45(1):57-64

8. Ismail R, Haq AH, Azman M, Rahman AF. Therapeutic drug monitoring of gentamicin: a 6-year follow-up audit. J Clin Pharm Ther. 1997;22(1):21-25.

9. Saunders NJ, Adams DJ, Lynn WA. A prospective laboratory-based audit of gentamicin use and therapeutic monitoring J Antimicrob Chemother. 1995;36(4):729-736.

10. Rybak MJ, Abate BJ, Kang SL, Ruffing MJ, Lerner SA, Drusano GL. Prospective evaluation of the effect of an aminoglycoside dosing regimen on rates of observed nephrotoxicity and ototoxicity. Antimicrob Agents Chemother. 1999;43(7):1549-1555.

11. Ho KK, Thiessen JJ, Bryson SM, Greenberg ML, Einarson TR, Leson CL. Challenges in comparing treatment outcome from a prospective with that of a retrospective study: assessing the merit of gentamicin therapeutic drug monitoring in pediatric oncology. Ther Drug Monit. 1994;16(3):238-247.

12. Slaughter RL, Cappelletty DM. Economic impact of aminoglycoside toxicity and its prevention through therapeutic drug monitoring. Pharmacoeconomics. 1998; 14: 385-394.

13. Rougier F, Ducher M, Maurin M, Corvaisier S, Claude D, Jelliffe R, Maire P. Aminoglycoside dosages and nephrotoxicity: quantitative relationships. Clin Pharmacokinet. 2003;42(5):493-500.

14. Goldhaber-Fiebert JD, Denny LE, De Souza M, Wright TC, Kuhn L, Goldie SJ. The costs of reducing loss to follow-up in South African cervical cancer screening. Cost Eff Resour Alloc. 2005;3:11.

15. Ab Rahman AF, Abdelrahim HEA, Mohamad Ibrahim MI. A survey of therapeutic drug monitoring services in Malaysia. Saudi Pharm J. 2013;21(1):19-24. doi: 10.1016/j.jsps.2012.01.002

16. Gross AS. Best practice in therapeutic drug monitoring. Br J Clin Pharmacol. 2001;52(Suppl 1):5S-10S.

17. Hammett-Stabler CA, Johns T. Laboratory guidelines for monitoring of antimicrobial drugs. National Academy of Clinical Biochemistry. Clin Chem. 1998;44(5):1129-1140.

18. Begg EJ, Barclay ML, Kirkpatrick CM. The therapeutic monitoring of antimicrobial agents. Br J Clin Pharmacol. 2001;52(Suppl 1):35S-43S.

19. Martin JE. Commentary: once-daily aminoglycoside dosing: where are we now? J Crit Care. 2003;18(2):113-114. 